\title{
PAPER
}

\section{Oropharyngeal swallowing in craniocervical dystonia}

\section{Ertekin, I Aydogdu, Y Seçil, N Kıylıoglu, S Tarlacı, T Ozdemirkıran}

J Neurol Neurosurg Psychiatry 2002;73:406-411

See end of article for authors' affiliations ......................

Correspondence to: Prof DrC Ertekin, Gönc Apt Talatpașa Bulvarı, No 12, Daire 335220

Alsancak, Izmir, Turkey; erteker@unimedya.net.tr

Received

3 December 2001

In revised form 4 April 2002

Accepted 6 June 2002

\begin{abstract}
Objective: To clarify the pathophysiology of dysphagia by electrophysiological methods. Method: Electrophysiological methods related to oropharyngeal swallowing were used to investigate 25 patients with cervical dystonia and 25 age matched normal volunteers.

Results: Dysphagia was suspected in $36 \%$ of patients with cervical dystonia on the basis of clinical assessment. The incidence of dysphagia increased to $72 \%$ on electrophysiological evaluation of pharyngeal swallowing. Submental muscle electromyographic (EMG) and laryngeal relocation times were significantly prolonged and the triggering time to swallowing reflex was significantly delayed. Some abnormalities seen in cricopharyngeal sphincter muscle EMG indicated that the striated sphincter muscle is hyperreflexive in some patients.

Conclusion: Neurogenic dysphagia was more prominent and longer lasting than mechanical dysphagia, which was transient and varied from patient to patient. Although these electrophysiological methods were not suitable for detecting anatomical changes during swallowing, as in videofluoroscopic studies, observations supported the neurogenic cause of dysphagia in patients with any kind of cervical dystonia.
\end{abstract}

$\mathrm{V}$ arious studies have shown that a range of swallowing difficulties accompany cervical dystonias such as spasmodic dysphonia (dystonia) ${ }^{12}$ and spasmodic torticollis before focal injections of the botulinum A toxin. ${ }^{3-5}$

Similar observations have been documented before and after cranial neurectomies ${ }^{67}$ and $\mathrm{Cl}-\mathrm{C} 3$ rhizotomies $^{89}$ in the above two types of cervical dystonias.

Two possible mechanisms have been proposed for dysphagia in patients with cervical dystonias before treatment with either botulinum toxin type A injection or surgical intervention. One is the abnormal position of the neck leading to anatomical asymmetry of swallowing. ${ }^{3910}$ However, this interpretation does not account for the dysphagia encountered in laryngeal or some oromandibular dystonias because of the lack of associated abnormal neck movements in most. The second possibility is a neurogenic cause that leads to a delayed swallowing initiation and other oropharyngeal findings. ${ }^{35910}$

The function of the oropharynx have been commonly investigated by videofluoroscopy except in one study. ${ }^{1}$ Horner et $a l^{8}$ have also used videofluoroscopy but proposed that electromyographic (EMG) methods may be useful in elucidating the pathophysiology of dysphagia in torticollis.

To clarify the role of mechanical or neurogenic dysphagia associated with cervical dystonia we investigated 25 patients with cervical dystonias of different types by using electrophysiological methods described previously. ${ }^{112}$

\section{METHOD}

\section{Patients}

Twenty five patients with cervical dystonia (13 male and 12 female) were investigated. The average duration of illness ranged from a few months to many years. Their average age was 42.8 years (range 19-68). Patients were divided into three groups according to their clinical presentations:

- Pure spasmodic torticollis group: 11 patients with leftward pulling in 3, rightward pulling in 6, and anterocollis in 2 .

- Oromandibular and laryngeal dystonia group: 7 patients with spastic dysphonia in 3, oromandibular dystonia in 3, and lingual dystonia in 1 . One patient with oromandibular dystonia also had anterolaterocollis but the others had no torticollis at all.
- Generalised dystonia plus spasmodic torticollis: 7 patients.

\section{Method}

Oropharyngeal function was clinically evaluated in each patient according to our simple grading of dysphagia. ${ }^{13}$ This classification is based purely on symptoms and signs of difficulty swallowing. The degree of dysphagia was graded as follows:

- grade 1: no clinical signs or symptoms of dysphagia were present.

- grade 2: very mild dysphagia was suspected in clinical examination but the patient never complained directly of dysphagia.

- grade 3: the patient complained of dysphagia and this was supported by other clinical signs.

- grade 4: obvious clinical signs and symptoms of dysphagia including aspiration; dysphagia severe enough to necessitate non-oral feeding.

The first group of patients was investigated either just before the first injection of botulinum A toxin or over the two months after the last botulinum A toxin injection. The remaining patients were not given any chemodenervation therapy or surgical intervention.

\section{Electrophysiological evaluation of oropharyngeal swallowing}

EMG methods to investigate the nature of oropharyngeal swallowing have been previously published. ${ }^{11}{ }^{12}$

Firstly, the onset and duration of oropharyngeal swallowing were recorded from the submental or suprahyoid muscles (SM-EMG). SM-EMG surface electrodes were usually placed at the midline bilaterally under the chin.

Secondly, the vertical movements of the larynx during swallowing were monitored by a piezoelectric sensor placed between the thyroid and the cricoid cartilages. This supplied

Abbreviations: $C P$, cricopharyngeal; $E M G$, electromyographic; $S C M$ sternocleidomastoid; SM, submental 


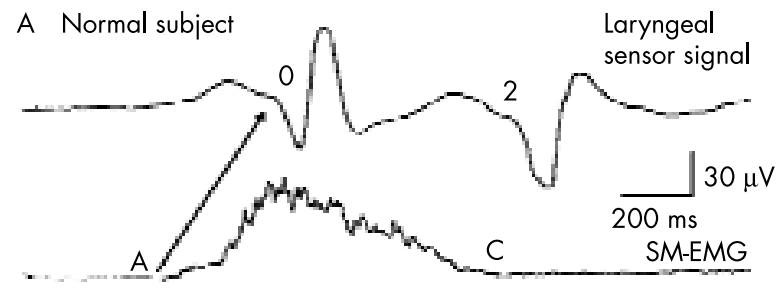

B Torticollis

Figure 1 Laryngeal sensor signals (upper traces in $A$ and $B$ ) and integrated submental electromyographic (SM-EMG) activities (lower traces in $A$ and $B$ ) obtained from a normal subject (A) and a patient with torticollis (B). Points $A$ and $C$ denote the onset and end of SM-EMG activity. Points 0 and 2 denote the onsets of laryngeal upward and downward movement, respectively. The oblique arrow from A to 0 (A-O interval) denotes the time interval from the onset of voluntarily triggered swallowing to the onset of reflex swallowing as shown by the onset of laryngeal upward movement. Extreme prolongation of the A-C interval and a delay in triggering time of the swallowing reflex (oblique arrow) are observed in the patient (B). Amplitude calibration is 30 and $50 \mu \mathrm{V}$, respectively, for SM-EMG traces. Time calibration is $200 \mathrm{~ms}$ all traces. All traces are averages of five responses.

data about the timing of laryngeal elevation and suspension during the pharyngeal phase of the reflex response. ${ }^{114}$

The total duration of SM-EMG activity was determined from the A-C interval and the laryngeal relocation time was measured from the onsets of the upward and downward signals of the laryngeal sensor, or the 0-2 time interval, during swallowing (fig l).

The triggering time of the pharyngeal phase or reflex swallowing was calculated from the time interval between the onsets of SM-EMG and the first deflection of the laryngeal sensor movement (the A-0 interval in fig 1).

Thirdly, using the same EMG methods described above, it was possible to diagnose objectively the existence of dysphagia in suspected cases by an indicator we called the dysphagia limit. While $3 \mathrm{ml}$ of water was swallowed in single bolus analysis, the dysphagia limit was determined by increasing the amount of water swallowed step by step from 3 to $20 \mathrm{ml}$ of water. The analysis time was taken as 10 seconds for this purpose instead of the 2 or 5 seconds allowed for single bolus analysis. Normal subjects can swallow a $20 \mathrm{ml}$ bolus of water in a single attempt. ${ }^{12}{ }^{15}$ Dysphagic patients are unable to swallow $20 \mathrm{ml}$ water volumes at one go and divide the bolus into two or more parts within 8 seconds. ${ }^{12}{ }^{15}$ All patients were investigated by this method in a sitting position with or without their head being held by one of the examiners.

Fourthly, in nine patients, EMG activity of the cricopharyngeal (CP) muscle of the upper oesophageal sphincter was recorded through a concentric needle electrode inserted percutaneously and directed posteromedially in the neck. ${ }^{11-13}$

The individual results obtained from the 25 patients with cervical dystonia were compared with the upper limits of deglutition parameters obtained from 75 normal adult subjects $^{12}$ divided into the three age groups of 17-39 years, 40-59 years, and 60-81 years. The individual values of CP sphincter EMG during swallowing were also compared with the normal values obtained from 25 normal age matched adult subjects with a mean age of 42.2 years (range 17-69, 13 male, 12 female). Informed consent was obtained from all patients and normal subjects. The investigation was approved by the ethical committee of our university hospital.

\section{RESULTS}

\section{Clinical assessment}

In the clinical assessment, 9 of 25 patients with cervical dystonia had expressed some subjective complaints or had objective clinical signs suggesting oropharyngeal dysphagia. Swallowing problems were mild in the spasmodic torticollis and the oromandibular and laryngeal dystonia groups and only two patients had grade 2 dysphagia. On the other hand, five of seven patients with generalised dystonia plus cervical dystonia had trouble swallowing and their degree of dysphagia changed from grade 2 to grade 3 . Thus, the proportion with dysphagia was $36 \%$ among patients with cervical dystonia based on clinical assessment. The dysphagia was mild and some patients compensated for their swallowing problems by special head and neck manoeuvres while swallowing. The majority of patients did not complain of any kind of deglutition problems. Despite this lack of complaints, the electrophysiological evaluations showed that "subclinical dysphagia" is not uncommon and precedes medical or surgical treatment.

\section{Dysphagia limit}

Figure 2 illustrates the dysphagia limits of one patient with and one without clinical dysphagia. Non-dysphagic patients swallowed up to $20 \mathrm{ml}$ of water in a single attempt, whereas patients with dysphagia divided the bolus into two or more parts while trying to swallow $20 \mathrm{ml}$ or less of water. A dysphagia limit of $<20 \mathrm{ml}$ of water was found not only in dysphagic patients but also in others with normal swallowing (dysphagia grade 1). The abnormal dysphagia limit was encountered in 18 patients, making the frequency $f$ dysphagia $72 \%$ in patients with cervical dystonia. Thus, subclinical dysphagia was determined in $36 \%$ of all patients with cervical dystonia by using these electrophysiological methods. The dysphagia limit was often $15-20 \mathrm{ml}$ water in patients with cervical dystonia with subclinical dysphagia, whereas patients with clinical dysphagia were not able to swallow $1-10 \mathrm{ml}$ in one attempt. The dysphagia limit was also useful to understand the effect of neck movements on swallowing. Some patients prevent the effect of sudden neck movements by fixing the head or changing the position to anteflexion during swallowing. In such patients, having the head and neck held by the examiner improved their dysphagia limit, as fig 3 illustrates. Unfortunately, the effect of holding the head could not be investigated in all patients because of difficulty in obtaining physical cooperation.

\section{Single bolus analysis}

Table 1 shows individual results of the single bolus analysis of swallowing $3 \mathrm{ml}$ of water. The most frequent and suggestive finding was the significant prolongation of SM muscle complex activity (A-C interval in table 1 and fig 1 ). The age dependant increase in the duration of SM muscle activity in the patients was accounted for by comparison with three age groups of normal subjects (17-39, 40-59, and 60-81 years, published previously ${ }^{12}$ ). The abnormal prolongation of SM muscle complex activity during $3 \mathrm{ml}$ water swallowing was found in 17 patients, mostly with generalised dystonia plus torticollis. This may mean that $68 \%$ of patients with cervical dystonia have abnormally high and long firing of the motor 
A Torticollis without dysphagia

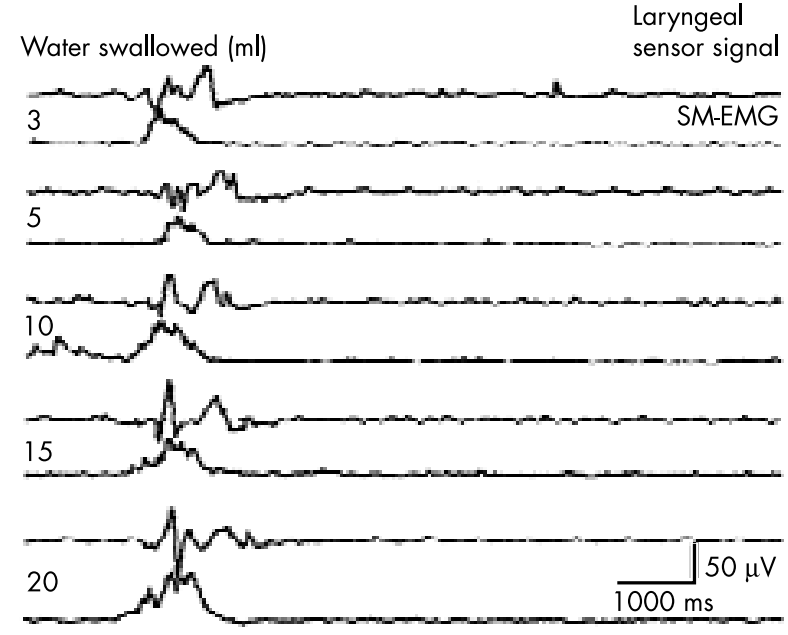

B Spasmodic dysphonia with dysphagia

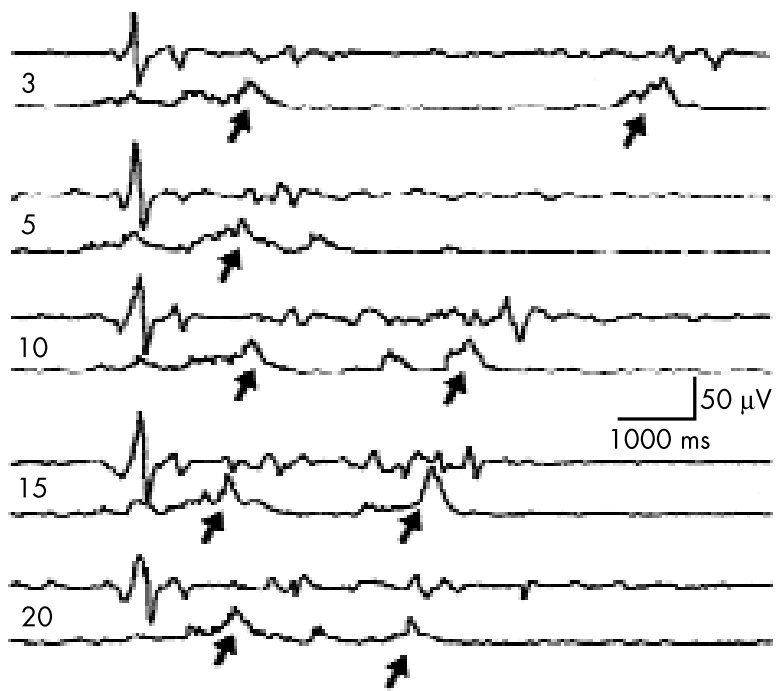

Figure 2 Laryngeal sensor signals (top traces in $A$ and $B$ ) and integrated SM-EMG activities (lower traces in A and B) obtained from two patients with cervical dystonia during swallowing of an increasing amount of water, from 3-20 ml. The non-dysphagic patient with torticollis was able to swallow up to $20 \mathrm{ml}$ in a single attempt, while the patient with spasmodic dysphonia with dysphagia divided the 3-20 ml boluses into two or more swallows. The dysphagia limit was $3 \mathrm{ml}$ water in the patient with spasmodic dysphonia. Arrows mark the second and following swallows. Amplitude calibration is $50 \mu \mathrm{V}$ for SM-EMG traces. Time calibration is $1000 \mathrm{~ms}$ in all traces. (Amplitude of laryngeal sensor signal is irrelevant.)

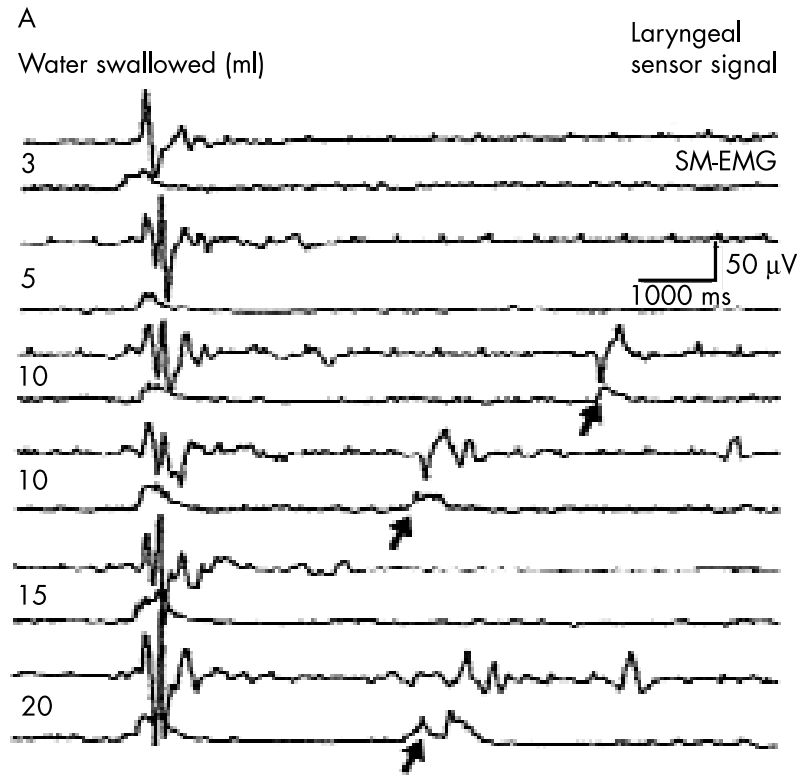

B

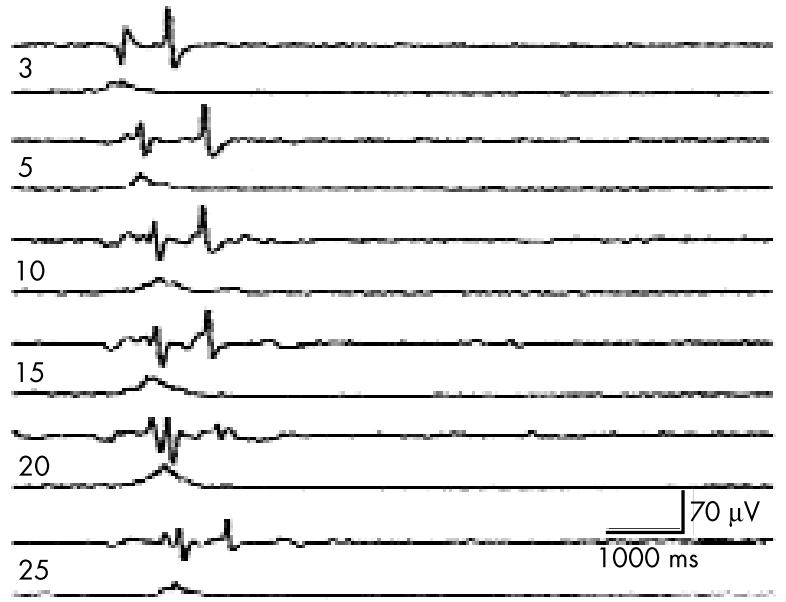

Figure 3 Laryngeal sensor signals (top trace in A and B) and integrated SM-EMG activities (lower traces in A and B) obtained from a patient with torticollis with the patient's head and neck in the laterocollis position (A) and with the head held by the examiner in neutral position (B) during swallowing of 3-20 ml of water. The volume swallowed rose to $25 \mathrm{ml}$ while in the neutral position (B), whereas in the laterocollis position (A) the patient divided the 10 and $20 \mathrm{ml}$ boluses into two swallows. Arrows mark the second swallows. Amplitude calibration is 50 (A) and $70 \mu \mathrm{V}(\mathrm{B})$ for SM-EMG traces. Time calibration is $1000 \mathrm{~ms}$ in all traces.

units of the submental laryngeal elevator muscles. The results were also significant (mean (SEM) 1200.3 (63.3) ms $v 857.9$ (23.2) ms, p < 0.0001). The second common abnormality was prolongation of the laryngeal relocation time during swallowing (0-2 interval in table 1 and fig 1$)$. This finding was encountered in 10 of the 25 patients $(42 \%)$. This time interval is intimately related with the time required for the pharyngeal phase of the swallowing reflex. ${ }^{11} 13$ This result was also significant (674.1 (26.2) ms $v 571.5$ (14.2) ms, p < 0.0001). Lastly, the triggering time of the swallowing reflex was delayed in eight patients (A-0 interval in table 1 and fig 1 ). This was also significant in all patient groups (410.5 (48.1) ms $v 261.4$ (20.9) ms, p < 0.0001). Overall, $31 \%$ of patients exhibited this delay but the incidence was higher in patients with oromandibular and laryngeal dystonia (four of seven patients). Thus, one or more of these neurogenic signs of swallowing disorder ${ }^{12}$ were found in 19 patients (76\%) by single bolus analysis. 
Table 1 Individual results of electrophysiological findings of patients with cervical dystonia

\begin{tabular}{|c|c|c|c|c|c|c|c|}
\hline Patient & Age (years)/sex & $\begin{array}{l}\text { Dysphagia } \\
\text { grade }\end{array}$ & $\begin{array}{l}\text { Dysphagia limit } \\
\text { (ml water) }\end{array}$ & A-C interval (s) & 0-2 interval (s) & A-0 interval (s) & CP-EMG \\
\hline \multicolumn{8}{|c|}{ Spasmodic torticollis } \\
\hline 1 & $38 / F$ & 1 & 20 & 850 & 680 & 150 & ND \\
\hline 2 & $52 / \mathrm{M}$ & 1 & 20 & 1600 & 745 & 520 & Normal \\
\hline 3 & $41 / M$ & 1 & 10 & 1106 & 908 & 142 & ND \\
\hline 4 & $39 / M$ & 1 & Normal & 1174 & 700 & 454 & ND \\
\hline 5 & $52 / \mathrm{F}$ & 1 & Normal & 1150 & 630 & 450 & ND \\
\hline 6 & $62 / \mathrm{F}$ & 1 & Normal & 938 & 616 & 200 & ND \\
\hline 7 & $23 / \mathrm{F}$ & 2 & 1 & 1278 & 414 & 380 & ND \\
\hline 8 & $59 / \mathrm{F}$ & 1 & Normal & 1332 & 676 & 570 & ND \\
\hline 9 & $38 / \mathrm{F}$ & 2 & 10 & 1110 & 640 & 284 & Abnormal \\
\hline 10 & $35 / \mathrm{F}$ & 1 & Normal & 1050 & 530 & 320 & ND \\
\hline 11 & $36 / M$ & 1 & 10 & 1030 & 544 & 256 & ND \\
\hline \multicolumn{8}{|c|}{ Oromandibular/laryngeal dystonia } \\
\hline 12 & $62 / F$ & 1 & 20 & 1226 & 800 & 364 & Normal \\
\hline 13 & $50 / \mathrm{M}$ & 2 & 5 & 1790 & 625 & 1125 & ND \\
\hline 14 & $41 / M$ & 1 & 20 & 1955 & 585 & 555 & ND \\
\hline 15 & $32 / M$ & 2 & 3 & 1198 & 550 & 544 & Abnormal \\
\hline 16 & $45 / M$ & 1 & Normal & 802 & 650 & 124 & ND \\
\hline 17 & $52 / M$ & 1 & 15 & 900 & 545 & 300 & ND \\
\hline 18 & $69 / \mathrm{F}$ & 1 & 20 & 940 & 590 & 600 & ND \\
\hline \multicolumn{8}{|c|}{ Generalised dystonia plus spasmodic torticollis } \\
\hline 19 & $55 / \mathrm{M}$ & 1 & 20 & 892 & 524 & 336 & Abnormal \\
\hline 20 & $19 / \mathrm{F}$ & 3 & 1 & 1068 & 712 & 170 & Abnormal \\
\hline 21 & $35 / M$ & 3 & 5 & ND* & 872 & $N D^{*}$ & Abnormal \\
\hline 22 & $22 / M$ & 2 & 10 & 1835 & 755 & 890 & Abnormal \\
\hline 23 & $22 / F$ & 1 & Normal & 1104 & 922 & 278 & ND \\
\hline 24 & $32 / M$ & 2 & 15 & 1255 & 845 & 280 & Abnormal \\
\hline 25 & $60 / \mathrm{F}$ & 2 & 5 & 1225 & 795 & 495 & ND \\
\hline \multicolumn{8}{|c|}{ Upper limit in normal controls ${ }^{12}$ by age group (years) } \\
\hline $17-39$ & & & $>20$ & 1064 & 726 & 495 & \\
\hline $40-59$ & & & $>20$ & 986 & 702 & 520 & \\
\hline $60-81$ & & & $>20$ & 1250 & 688 & 492 & \\
\hline
\end{tabular}

F, female; M, male; ND, Not done. *Submental muscle electromyography (SM-EMG) could not be measured because of severe muscle and movement artefacts.

\section{CP sphincter EMG}

The CP sphincter muscle of the pharyngoesophageal segment could be investigated in only nine patients. Among these patients CP-EMG during swallowing was found to be normal in two patients who had a "CP-EMG pause" lasting 400-600 ms and with forebursts and rebound bursts of CP tonic activity (fig 4). However, the remaining seven patients (one with oromandibular and laryngeal dystonia, one with spasmodic torticollis, and the rest with generalised dystonia) were found to have some abnormalities indicating a kind of hyperreflexion of the sphincter muscle..$^{12}{ }^{16}$ Among these, the unexpected burst of motor units within the CP-EMG pause was common (fig 4). Other less frequent abnormalities encountered were shortening of the pause and premature closure of the CP sphincter EMG. It was noted that the absence of the foreburst was also quite frequent in patients with cervical dystonia but not usually observed in hyperreflexive $\mathrm{CP}$ sphincter. $^{1316}$

\section{Relation between swallowing and sternocleidomastoid muscle activity}

In four patients with spasmodic torticollis, the SM-EMG and the hyperreflexive sternocleidomastoid (SCM) EMG (needle recording) were recorded synchronously. There was no swallowing activation as traced by SM-EMG and laryngeal sensor during the SCM burst activity due to clinical spasms.

Similarly, the swallowing movement did not produce any EMG activation in the SCM muscle. During the dysphagia limit investigation with boluses from 3 to $20 \mathrm{ml}$ of water, in all four cases the second swallow was not synchronous with SCM activation. Some patients had discovered special head or neck positions and holding the head in a stable position sometimes improved swallowing (fig 3). Mechanical dysphagia seemed to be a transient phenomenon. In other patients with clinical dysphagia, holding the head or neck fast or maintaining a lat- eral spine resting position did not prevent repetitive swallowing or improve the dysphagia limit, though these procedures were not performed in all patients.

Summing up, involuntary neck movements did not continuously produce dysphagic signs clinically or electrophysiologically; nor did swallowing aggravate the head or neck positions in our patient groups.

\section{DISCUSSION}

Dysphagia was suspected in $36 \%$ of patients with cervical dystonia on the basis of clinical assessment. The incidence of dysphagia increased to $72 \%$ on electrophysiological evaluation of oropharyngeal swallowing.

Clinical and videofluoroscopic evaluations have also indicated a high incidence of swallowing disorders in patients with cervical dystonia ${ }^{389}$ before any treatment such as botulinum toxin injection or rhizotomy. The incidence of dysphagia varied between 22 and 100\% of patients (mostly over 50\%). The incidence of dysphagia increased more significantly after botulinum toxin injection ${ }^{1017-19}$ and after selective rhizotomy. ${ }^{89}$ Similarly, dysphagia in patients with spasmodic dysphonia has been reported before and after treatment of this condition. ${ }^{267}$

In our patient groups 11 had pure spasmodic torticollis and 7 had torticollis with generalised dystonia. However, in our 7 patients with oromandibular and laryngeal dystonia there were no abnormal neck movements except in one patient. Despite the heterogeneity of the patients with cervical dystonia, the clinical and electrophysiological findings were similar for each group.

The major finding of this study was that the neurogenic nature of dysphagia was more prominent and longer lasting than the mechanical dysphagia, which was transient and varied from patient to patient. Although our electrophysiological 


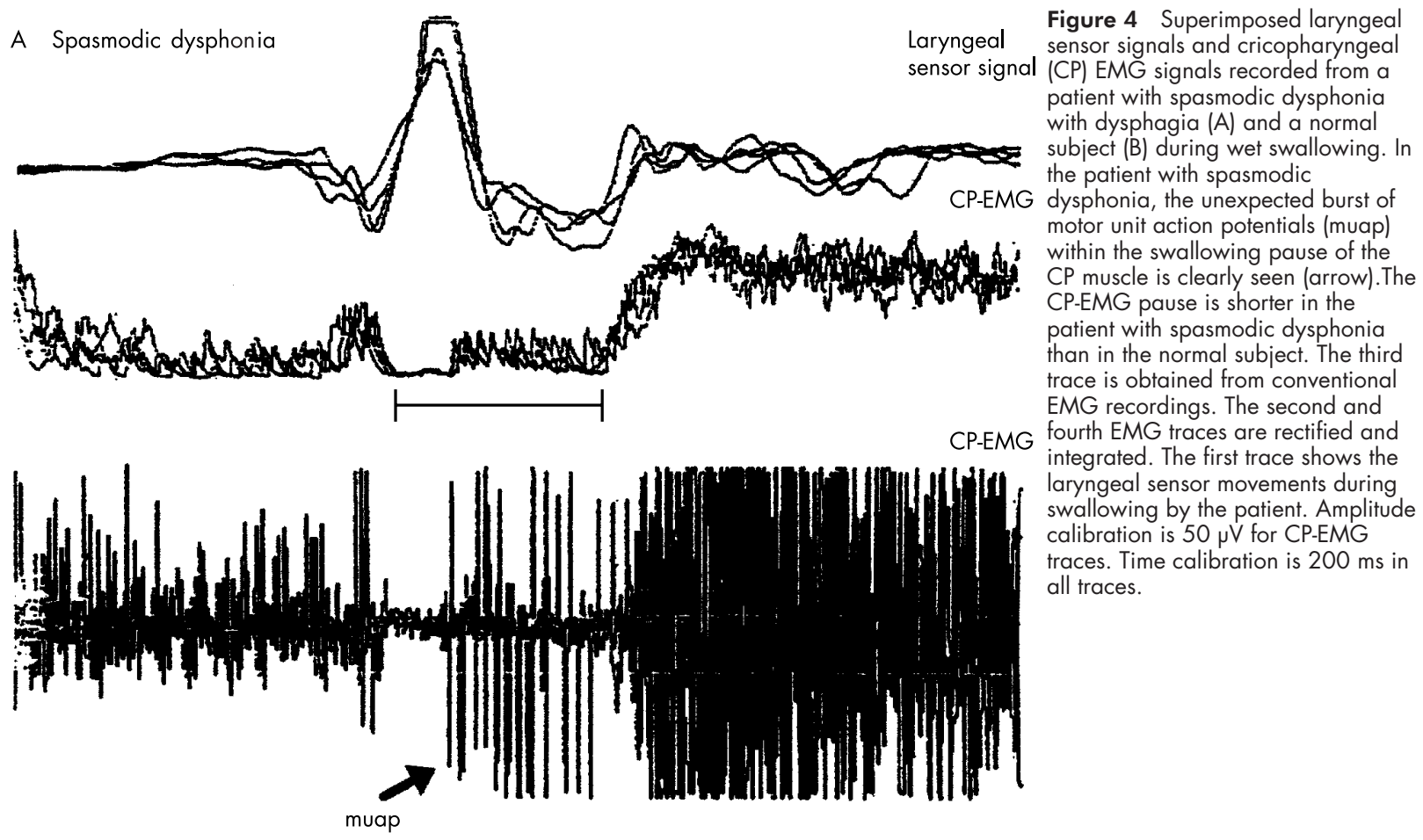

B Normal subject

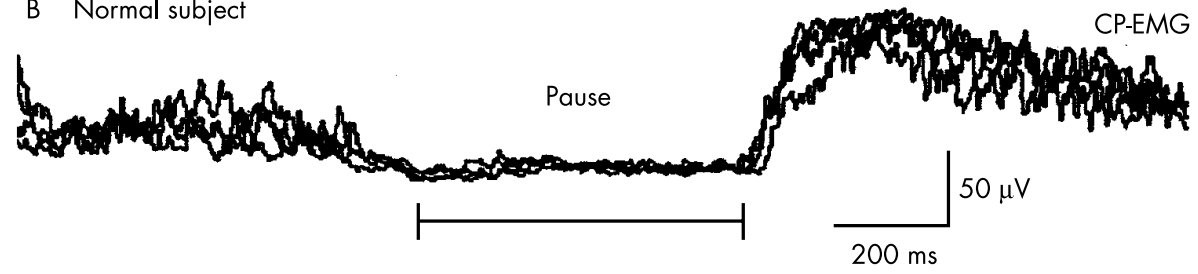

methods were not suitable for detecting anatomical changes during swallowing, as in videofluoroscopic studies, the following observations supported the neurogenic cause of dysphagia in patients with any kind of cervical dystonia:

Firstly, similar clinical and electrophysiological findings were found in three groups of patients including the oromandibular and laryngeal dystonia group, who did not have spasmodic torticollis. Therefore, head or neck movement is not essential for dysphagia

Secondly, some patients maintained a certain position to compensate for the adverse effect of neck movement during swallowing. Holding or fixing the head was beneficial in some other patients (fig 3 ). In clinically affected patients and some other patients, stabilising the head and neck was not effective in changing the patients' dysphagia clinically, nor were the dysphagia limits improved.

Thirdly, no EMG relation was observed between swallowing and EMG activity of the SCM muscle.

Fourthly, electrophysiological abnormalities were frequent and all were pertinent to neurogenic dysphagia. ${ }^{12}{ }^{13}$ Videofluoroscopic evaluation of patients with cervical dystonia generally has shown delayed initiation of swallowing and pharyngeal residue. ${ }^{39}$ These abnormalities are regarded as neurogenic dysfunction in oropharyngeal assessment with videofluoroscopy. ${ }^{20}$ In our patients the most frequently encountered electrophysiological abnormality was prolongation of SM muscle complex activity during swallowing ( $68 \%$ of all patients). Though prolongation of the laryngeal relocation time and triggering of the swallowing reflex were less frequent than SM muscle activity, they were seen in $42 \%$ and $31 \%$, respectively, of all patients with cervical dystonia. Such findings are observed mostly in patients with Parkinson's disease. ${ }^{12}$ They are not related to mechanical dysphagia. In normal subjects, when the head is rotated towards any side or held in retroflexion, the dysphagia limit may change but the single bolus parameters of the SM-EMG complex and sensor recording never change. ${ }^{2}$

Fifthly, CP sphincter EMG during swallowing was not normal in patients with cervical dystonia, especially in those in the generalised dystonia group. Some of the CP-EMG findings were compatible with the hyperreflexive/hypertonic nature of the upper oesophageal sphincter ${ }^{13}{ }^{16}$ observed in suprabulbar involvement of the bulbar swallowing centre in the central nervous system.

Lastly, aggravation of dysphagia following medical and surgical treatment of spasmodic torticollis points to the neurogenic nature of dysphagia; otherwise, an improved head or neck position would have eased the mechanical dysphagia. Botulinum toxin treatment and rhizotomies may increase dysphagic signs or unmask the potential inherent neurogenic nature of dysphagia in these patients. However, transient brisk neck spasms may still produce a transient and sporadic negative effect on swallowing at any time.

It is not known how and where the neurogenic dysphagia of cervical dystonia is located in the central nervous system. This awaits further studies in line with the pathophysiology of the general nature of dystonias.

\section{Authors' affiliations}

${ }^{*}$ C Ertekin, **I Aydogdu, Y Seçil, N Kıylıoglu, S Tarlacı, T Ozdemirkıran, Department of Neurology, Ege University Medical School Hospital, Bornova, Izmir, Turkey

*Also the Department of Clinical Neurophysiology, Ege University Medical School Hospital, Bornova, and The Centre of Clinical Neurophysiology and EMG, Alsancak, Izmir, Turkey 
**Also the Department of Clinical Neurophysiology, Ege University Medical School Hospital, Bornova, Izmir, Turkey

Competing interests: none declared

\section{REFERENCES}

1 Holzer SE, Ludlow CL. The swallowing side effects of botulinum toxin type $\mathrm{A}$ injection in spasmodic dysphonia. Laryngoscope 1996; 106:86-92.

2 Ludlow CL, Naunton RF, Sedary SE, et al. Effect of botulinum toxin injections on speech in adductor spasmodic dysphonia. Neurology 1988:38:1220-5.

3 Riski JE, Horner J, Nashold BS Jr. Swallowing function in patients with spasmodic torticollis. Neurology 1990;40: 1443-5.

4 Comella CL, Tanner CM, Defoor-Hill L, et al. Dysphagia after botulinum toxin injections for spasmodic torticollis: clinical and radiological findings. Neurology 1992:42:1307-10.

5 Horner J, Riski JE, Weber BA, et al. Swallowing speech and brainstem auditory evoked potentials in spasmodic torticollis. Dysphagia 1993;8:29-34

6 Dedo HH, Izdebski K. Problem with surgical (RLN section) treatment of spastic dysphonia. Laryngoscope 1983:93:268-71.

7 Aronson AE, De Santo LW. Adductor spasmodic dysphonia: three years after recurrent laryngeal nerve section. Laryngoscope 1983;93:1-8.

8 Horner J, Riski JE, Ovelman-Levitt J, et al. Swallowing in torticollis before and after rhizotomy. Dysphagia 1992;7:117-25.

9 Münchau A Good CD, McGowan S, et al. Prospective study of swallowing function in patients with cervical dystonia undergoing selective peripheral denervation. J Neurol Neurosurg Psychiatry $2001 ; 71: 67-72$
10 Whurr R, Bhatia KP, Masarei A, et al. The incidence and nature of dysphagia following botulinum toxin injections for torticollis: a prospective study of 123 patients. J Med Speech Lang Pathol 1999;7:196-207.

11 Ertekin C, Pehlivan M, Aydogdu I, et al. An electrophysiological investigation of deglutition in man. Muscle Nerve 1995:18:1177-86.

12 Ertekin C, Aydogdu I, Yuceyar N, et al. Electrodiagnostic methods for neurogenic dysphagia. Electroencephalogr Clin Neurophysiol 1998; 109:331-40.

13 Ertekin C, Aydogdu I, Yüceyar N, et al. Pathophysiological mechanisms of oropharyngeal dysphagia in amyotrophic lateral sclerosis. Brain 2000:1 23: 125-40.

14 Pehlivan M, Yüceyar N, Ertekin C, et al. An electronic device measuring the frequency of spontaneous swallowing: digital phagometer. Dysphagia 1996;11:255-64.

15 Ertekin C, Aydogdu I, Yüceyar N. Piecemeal deglutition and dysphagia limit in normal subjects and patients with swallowing disorders. J Neurol Neurosurg Psychiatry 1996;61:491-6.

16 Ertekin C, Aydogdu I, Tarlaci S, et al. Mechanisms of dysphagia in suprabulbar palsy with lacunar infarct. Stroke 2000;31:1370-6.

17 Borodic GE, Joseph M, Fay L, et al. Botulinum A toxin for the treatment of spasmodic torticollis: dysphagia and regional toxin spread. Head Neck 1990;12:392-8.

18 Jankovic J, Schwartz K. Response and immuneresistance to botulinum toxin injections. Neurology 1995;45: 1743-6.

19 Blackie JD, Lees AJ. Botulinum toxin treatment in spasmodic torticollis. J Neurol Neurosurg Psychiatry 1990;53:640-3.

20 Logemann JA. Dysphagia in movement disorders. Adv Neurol 1988:49:307-16.

21 Ertekin C, Keskin A, Kiylioglu N, et al. The effect of head and neck positions on oropharyngeal swallowing: a clinical and electrophysiologic study. Arch Phys Med Rehabil 2001;82:1255-60.

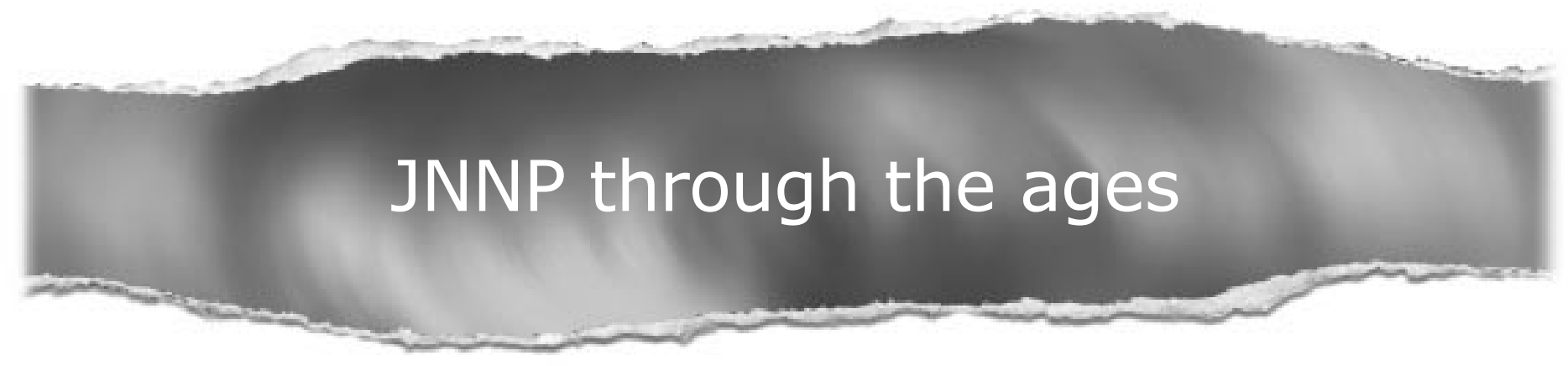

Browse the Archive

Journal of Neurology, Neurosurgery, and Psychiatry online has an archive of content dating back to 1966. Full text from 1997; abstracts from 1975; table of contents from 1966

www.jnnp.com 